\title{
Primary and combined resistance to four antimicrobial agents in Helicobacter pylori in Sofia, Bulgaria
}

\author{
LUDMILA BOYANOVA, IRINA STANCHEVA*, ZOYA SPASSOVA $\uparrow$, NIKOLAI KATZAROV $\uparrow$, IVAN \\ MITOV and RADKA KOUMANOVA
}

Department of Microbiology, Medical University, Sofia, *Department of Gastroenterology, Hospital of the Ministry of Internal Affairs, Sofia, †Department of Gastroenterology, University Hospital 'St Ivan Rilski', Sofia and $\$$ Department of Gastroenterology, University Paediatric Hospital, Sofia, Bulgaria

\begin{abstract}
The aim of this study was to evaluate the primary and combined resistance of Helicobacter pylori against four antimicrobial agents by a screening agar method (SAM) and a modified disk diffusion method (MDDM) alone and in combination. Pre-treatment $H$. pylori isolates from 192 consecutive $H$. pylori-positive patients at three hospitals in Sofia were investigated. MDDM was performed with disks containing metronidazole (5 $\mu \mathrm{g})$, clarithromycin (15 $\mu \mathrm{g})$ or erythromycin $(15 \mu \mathrm{g})$, ciprofloxacin $(5 \mu \mathrm{g})$ and tetracycline $(30 \mu \mathrm{g})$. Resistance was determined by an inhibitory zone of $<16 \mathrm{~mm}$ for metronidazole and $\leqslant \mathbf{3 0} \mathbf{m m}$ for other agents tested. The cut-off concentrations used to define resistance by SAM were: metronidazole $>8 \mathrm{mg} / \mathrm{L}$, clarithromycin $>2 \mathrm{mg} / \mathrm{L}$, tetracycline $>4 \mathrm{mg} / \mathrm{L}$ and ciprofloxacin $>1 \mathrm{mg} / \mathrm{L}$. Primary resistance rates in $\mathrm{H}$. pylori were: metronidazole $28.6 \%$, clarithromycin $9.7 \%$, metronidazole + clarithromycin $\mathbf{2 . 8} \%$, ciprofloxacin $3.9 \%$, metronidazole + ciprofloxacin $2.3 \%$, tetracycline $1.9 \%$ and metronidazole + tetracycline $1.2 \%$. Among metronidazole-resistant isolates, combined resistance to clarithromycin, ciprofloxacin and tetracycline was present in $11.4 \%$ (5 of 44 strains), $8.3 \%$ (3 of 36 ) and $4.9 \%$ ( 2 of 41 ), respectively. Two strains exhibited triple resistance to macrolides, metronidazole and either ciprofloxacin or tetracycline. Three tetracycline-resistant strains were detected in 1999; however, resistance rates to other agents were relatively stable during the 6 years. Primary $H$. pylori resistance to metronidazole is moderate and resistance to clarithromycin and to ciprofloxacin is considerable in comparison with results in most other countries. The alarming appearance of strains harbouring combined resistance or multiresistance provides the motivation for continued surveillance of $\mathrm{H}$. pylori at global, national and regional levels.
\end{abstract}

\section{Introduction}

Helicobacter pylori infection causes chronic gastritis, which can trigger peptic ulcer disease and gastric malignancies in man [1]. Successful eradication of the infection results in ulcer healing and prevents ulcer recurrences and complications [2]. Primary resistance in $H$. pylori has been reported to nitroimidazoles (in $6-95 \%$ of isolates), macrolides $(0-17 \%)$, ciprofloxacin $(0-1 \%)$ and tetracycline $(0-6 \%)$ in different countries

Received 5 April 1999; revised version accepted 10 Oct. 1999.

Corresponding author Dr L. Boyanova.
[2-8]. Both primary and secondary resistances of the bacterium are major reasons for therapeutic failure and require constant monitoring [2,9]. For this purpose, several methods for routine susceptibility testing of $H$. pylori have been evaluated, including the $\mathrm{E}$ test, modified disk diffusion method (MDDM) and screening agar method (SAM), and the standardisation of procedures is gradually progressing $[9,10]$. However, there is a lack of interpretive category standards for both minimal inhibitory concentrations (MICs) and inhibitory zone diameters for resistance to many antibiotics in $H$. pylori. The aim of the present study was to assess the primary resistance rates of clinical isolates of $H$. pylori to four antimicrobial agents and the combined resistance of the bacterium to more than one drug. 


\section{Materials and methods}

\section{Patients and biopsy specimens}

H. pylori strains were obtained from gastric biopsy specimens of 192 consecutive $H$. pylori-positive patients (113 men, 79 women; average age 30.2 years, range 3-76 years) with peptic ulcers (77 patients) and chronic gastritis (115 cases). The specimens were collected from patients undergoing routine gastrointestinal tract endoscopy at three hospitals in Sofia from June 1993 to June 1999. None of the patients had been treated with metronidazole, macrolides, tetracyclines or fluoroquinolones for the eradication of $H$. pylori infection. The strains were isolated and identified by standard procedures as described previously [11], and susceptibility testing was performed by MDDM or SAM, and by both methods in 70 cases.

\section{Modified disk diffusion method (MDDM)}

H. pylori strains were inoculated on to Brucella Blood Agar plates (Oxoid, Basingstoke, or Merck, Darmstadt, Germany) with Isovitalex (Becton Dickinson, BBL Microbiology Systems, Cockeysville, MD, USA) $1 \%$. Colonies were suspended in sterile saline and adjusted to a density equal to McFarland turbidity standard 3-4. The suspensions were spread on to the plates with sterile cotton swabs and then disks containing metronidazole $(5 \mu \mathrm{g})$, clarithromycin $(15 \mu \mathrm{g})$, ciprofloxacin $(5 \mu \mathrm{g})$ or tetracycline $(30 \mu \mathrm{g})$ were added. The clarithromycin susceptibility of 34 isolates was tested by erythromycin disks $(15 \mu \mathrm{g})$ because of the crossresistance with all macrolides [4]. Fauchere [12] recently reported a $100 \%$ concordance between susceptibility to erythromycin and clarithromycin in $H$. pylori. The plates were incubated micro-aerobically (Campylobacter Gas Generating Kits BR 060A, Oxoid, or Campy Pak Plus, Becton Dickinson) at $37^{\circ} \mathrm{C}$ for 3 days. Resistance was determined by a zone of growth inhibition $<16 \mathrm{~mm}$ corresponding to an MIC $>8 \mathrm{mg} / \mathrm{L}$ for metronidazole [13], and zone diameters $\leqslant 30 \mathrm{~mm}$ for clarithromycin or erythromycin, ciprofloxacin and tetracycline [8]. Greater zones of complete growth inhibition indicated the presence of susceptible strains. The MDDM susceptibility tests were performed in duplicate and median diameters of growth inhibition were taken into account.

\section{Screening agar method (SAM)}

Two drops (c. $60 \mu \mathrm{l})$ of $H$. pylori suspensions prepared as above were inoculated on one-quarter of the surface of Brucella Blood Agar plates containing one of the following drugs: metronidazole $8 \mathrm{mg} / \mathrm{L}$, clarithromycin $2 \mathrm{mg} / \mathrm{L}$, ciprofloxacin $1 \mathrm{mg} / \mathrm{L}$ or tetracycline $4 \mathrm{mg} / \mathrm{L}$. Metronidazole and tetracycline were obtained from Sigma, clarithromycin from Abbott Laboratories (Chicago, IL, USA) and ciprofloxacin from Bayer Pharma (Sens, France). The plates were incubated microaerobically at $37^{\circ} \mathrm{C}$ for 3 days. If $H$. pylori growth appeared on the plate, the isolate was deemed to be resistant to the corresponding drug. Non-selective plates were used as a control of strain viability. If resistance was detected by either SAM or MDDM or by both methods, the strain was considered to be resistant to the corresponding antimicrobial agent.

\section{Statistical analysis}

Differences between patients with susceptible and resistant strains were assessed by the $\chi^{2}$ test with or without Yates's correction.

\section{Results}

Primary resistance rates in $H$. pylori were as follows: metronidazole $28.6 \%$, clarithromycin $9.7 \%$, ciprofloxacin $3.9 \%$ and tetracycline $1.9 \%$ (Table 1 ). There were no differences $(\mathrm{p}>0.1)$ in the antimicrobial resistance rates and diseases (peptic ulcer versus chronic gastritis) or different age groups (patients $<17$ years old versus patients $\geqslant 18$ years old). Although women more frequently harboured strains resistant to metronidazole (40.5\% versus $20.4 \%$ in men), clarithromycin $(11.4 \%$ versus $8.6 \%)$ and ciprofloxacin $(6.1 \%$ versus $2.5 \%)$, the differences were significant $(\mathrm{p}<0.01)$ only in regard to metronidazole.

Combined resistance to metronidazole and either macrolides or fluoroquinolones was found in five of

Table 1. Primary and combined resistance of $H$. pylori to antimicrobial agents

\begin{tabular}{lcc}
\hline Agents & $\begin{array}{c}\text { Number of } \\
\text { strains }\end{array}$ & $\begin{array}{c}\text { Number (\%) of } \\
\text { resistant strains }\end{array}$ \\
\hline Metronidazole & 192 & $55(28.6)$ \\
Clarithromycin & 175 & $17(9.7)$ \\
Ciprofloxacin & 129 & $5(3.9)$ \\
Tetracycline & 160 & $3(1.9)$ \\
Metronidazole + clarithromycin & 175 & $5(2.8)$ \\
Metronidazole + ciprofloxacin & 129 & $3(2.3)$ \\
Clarithromycin + ciprofloxacin & 121 & $2(1.6)$ \\
Metronidazole + clarithromycin + ciprofloxacin & 121 & $1(0.8)$ \\
Metronidazole + tetracycline & 160 & $2(1.2)$ \\
Metronidazole + clarithromycin + tetracycline & 160 & $1(0.6)$ \\
\hline
\end{tabular}


175 strains $(2.8 \%)$ and in three of 129 strains $(2.3 \%)$, respectively, and two of 160 isolates $(1.2 \%)$ were not susceptible to both metronidazole and tetracycline. Among metronidazole-resistant isolates, combined resistance to clarithromycin, ciprofloxacin and tetracycline was observed in $11.4 \%, 8.3 \%$ and $4.9 \%$ respectively (Table 2). Clarithromycin-resistant isolates were not susceptible to metronidazole, ciprofloxacin and tetracycline in $26.7 \%, 20.0 \%$ and $7.7 \%$, respectively. One strain exhibited multiple resistance to clarithromycin, metronidazole and ciprofloxacin. This strain was isolated from the gastric mucosa of a 56year-old woman with duodenal ulcer. One isolate that was resistant to tetracycline, clarithromycin and metronidazole came from a 25-year-old man with chronic gastritis.

The metronidazole resistance rate was stable, being $28.1 \%$ in $1993-1996$ and $27.3 \%$ in $1998-1999$ $(\mathrm{p}>0.20)$. Incidences of resistance to macrolides (8.2\% in 1993-1996 and $11.5 \%$ in 1997-1999) and to ciprofloxacin $(2.1 \%$ in $1993-1996$ and $9.1 \%$ in 1997-1999) showed a slight tendency to increase; however, the differences were not statistically significant $(\mathrm{p}>0.20)$. Tetracycline resistance was detected only in 1999.

\section{Discussion}

Resistance of $H$. pylori to antimicrobial agents is the main cause of treatment failure. Even a triple regimen (including amoxycillin, clarithromycin and omeprazole) has usually led to $H$. pylori eradication for $50 \%$ of patients infected with clarithromycin-resistant strains versus $>90 \%$ for those harbouring susceptible isolates $[3,9]$. The clinical relevance of metronidazole resistance detected in vitro is more controversial. In one study, a triple scheme using amoxycillin, metronidazole and lansoprazole has been associated with a $96 \%$ cure rate for isolates with metronidazole MICs $<2 \mathrm{mg} / \mathrm{L}$ versus $45 \%$ for those with MICs $\geqslant 16 \mathrm{mg} / \mathrm{L}$; however, other authors have reported better eradication rates with similar regimens $[3,9]$.

H. pylori is a fastidious and slow growing microaerophilic bacterium, and its susceptibility testing has specific requirements. For this purpose, the breakpoint method $(8 \mathrm{mg} / \mathrm{L})$ has been recommended for metronidazole and different techniques are applicable for macrolides [9]. Although it is generally accepted that clarithromycin $2 \mathrm{mg} / \mathrm{L}$ and metronidazole $8 \mathrm{mg} / \mathrm{L}$ correspond to the breakpoints for $H$. pylori resistance [13-16], MIC interpretive standards for other antimicrobial agents are still lacking. Moreover, several authors have proposed an intermediate category for $H$. pylori strains inhibited by metronidazole $8 \mathrm{mg} / \mathrm{L}$ or exhibiting zone diameters of $16-<21 \mathrm{~mm}$ [13]. According to another study, the metronidazole intermediate category has been proposed for isolates with inhibitory zone diameters of $20-26 \mathrm{~mm}$ (MIC 4$8 \mathrm{mg} / \mathrm{L}$ ) [14]. These studies emphasise that metronidazole susceptibility testing of this organism is a great challenge and the results must be interpreted with caution.

The present study used tetracycline $4 \mathrm{mg} / \mathrm{L}$ for SAM susceptibility testing because this concentration is recommended by the NCCLS for determining susceptible strains in anaerobic microbiology [17]. The cut-off level for ciprofloxacin-susceptible strains $(1 \mathrm{mg} / \mathrm{L})$ was chosen because sensitive $H$. pylori isolates have exhibited MICs $\leqslant 0.5 \mathrm{mg} / \mathrm{L}$ and resistant strains have shown ciprofloxacin MICs $>2 \mathrm{mg} / \mathrm{L}$, according to Megraud [9].

The disk diffusion method is usually considered inappropriate for slow-growing organisms, because the gradient of drug concentration in the agar after prolonged incubation is not reproducible [18]. However, this technique is applicable for testing the susceptibility of $H$. pylori to antibiotics when there is a clearly bimodal population (e.g., clarithromycin) [3]. Different authors [13-15] have also reported MDDM to be a reliable method for metronidazole susceptibility testing. In the present study strains were considered to be resistant to metronidazole by MDDM according to the interpretive criteria of Chaves et al. [13]. The narrow range of MICs and homogeneous susceptibility of $H$. pylori to clarithromycin, ciprofloxacin and tetracycline have been reported [10]; therefore, large inhibitory areas $(>30 \mathrm{~mm})$ seem to be most suitable for detecting isolates which are susceptible to all drugs except metronidazole.

A considerable difference has been observed between the rates of primary metronidazole resistance of $H$. pylori in developed (6-49\%) and developing (77$95 \%$ ) countries because of the extensive use of nitroimidazoles for treating parasitic diseases in the

Table 2. Combined resistance in primary resistant $H$. pylori isolates

\begin{tabular}{lllll}
\hline & \multicolumn{4}{c}{ Combined resistance (\%) to } \\
\cline { 2 - 5 } Strains resistant to & Clarithromycin & Ciprofloxacin & Metronidazole & Tetracycline \\
\hline Metronidazole & 5 of $44(11.4)$ & 3 of $36(8.3)$ & $\ldots$ & 2 of $41(4.9)$ \\
Clarithromycin & $\ldots$ & 2 of $10(20.0)$ & 4 of $15(26.7)$ & 1 of $13(7.7)$ \\
Ciprofloxacin & 2 of $4(50.0)$ & $\ldots$ & 3 of $5(60.0)$ & 0 of $5(0)$ \\
Tetracycline & 1 of $3(33.3)$ & NT & 2 of $3(66.7)$ & $\ldots$ \\
\hline
\end{tabular}

NT, not tested. 
latter countries [2-5]. In the present study, the prevalence was similar to the median rates in Europe, and encompassed c. $29 \%$ of the pre-treatment isolates. On the contrary, the rate of clarithromycin resistance was higher than that observed in Germany and the Netherlands $(1-2 \%)$ and was similar to that reported in France (10\%) [2, 3, 5, 6]. Megraud [9] has emphasised that in the case of resistance rates $<15 \%$ to clarithromycin and $<30 \%$ to metronidazole, these agents could be used in therapeutic regimens without in-vitro susceptibility testing of $H$. pylori. The present results demonstrated that primary $H$. pylori resistance to metronidazole is close to the crucial $30 \%$ and that to macrolides is also worrying.

Although ciprofloxacin is not a drug of choice in the therapy of $H$. pylori infection, its combination with amoxycillin could be considered as an alternative in case of combined resistance of the bacterium to the first-line antimicrobial agents [9]. In comparison with the low prevalence $(\leqslant 1 \%)$ of ciprofloxacin resistance in $H$. pylori in most studies $[3,7]$ the present study found a striking $(3.9 \%)$ resistance rate. Carbone et al. [19] have reported high prevalence of fluoroquinolone resistance in $H$. pylori as well, three of the 32 strains tested being resistant to ciprofloxacin $>5 \mathrm{mg} / \mathrm{L}$. In the present work, all isolates were uniformly susceptible to tetracycline except three resistant strains detected in 1999. Resistance rates to the other agents tested were relatively stable over the 6 years of the study. Primary antibiotic resistance rates exhibited no association with age and clinical diagnosis. A significantly higher incidence of metronidazole resistance was observed in women than in men, as reported previously [4].

Approximately $1-3 \%$ of the pre-treatment isolates exhibited double resistance to different drugs. Combined resistance to metronidazole and clarithromycin was often detected among macrolide-resistant isolates (c. $25 \%$ ) and affected around $10 \%$ of the metronidazole-resistant strains. Simultaneous primary resistance to these agents was reported in four of six clarithromycin-resistant isolates by van Zwet et al. [5], and such strains have been associated with therapy failure [2]. The problem concerning combined and multiple resistance in $H$. pylori is of utmost importance, as the efficacy of the current therapeutic regimens may be compromised. Moreover, the present study detected two pre-treatment isolates showing triple resistance to metronidazole, clarithromycin and either ciprofloxacin or tetracycline. Nakae et al. [20] found triple resistance in $14.3 \%$ and double resistance in $28.6 \%$ of $H$. pylori strains resistant to macrolides, fluoroquinolones or nitroimidazoles.

In conclusion, the present results emphasise the need to perform constant monitoring of $H$. pylori resistance patterns to antimicrobial agents at global, national and regional levels in order to counteract the tendencies in the evolution of resistance.

\section{References}

1. Marshall BJ. Helicobacter pylori. Am J Gastroenterol 1994; 89 8 Suppl: S116-S128.

2. Adamek RJ, Suerbaum S, Pfaffenbach B, Opferkuch W. Primary and acquired Helicobacter pylori resistance to clarithromycin, metronidazole, and amoxicillin - influence on treatment outcome Am J Gastoenterol 1998; 93: 386-389.

3. Mégraud F. Resistance of Helicobacter pylori to antibiotics. Aliment Pharmacol Ther 1997; 11 Suppl 1: 43-53.

4. Mégraud F. Helicobacter pylori resistance to antibiotics. In: Hunt RH, Tytgat GNJ (eds) Helicobacter pylori: basic mechanisms to clinical cure. Dordrecht, Kluwer Academic. 1994: 570-583.

5. van Zwet AA, de Boer WA, Schneeberger PM, Weel J, Jansz AR, Thijs JC. Prevalence of primary Helicobacter pylori resistance to metronidazole and clarithromycin in the Netherlands. Eur J Clin Microbiol Infect Dis 1996; 15: $861-864$.

6. Tillenburg B, Siehoff S, Becker T, Peitz U, Borsch G, Labenz J. [Helicobacter pylori: therapeutic resistance status in Germany (Ruhr area).] Helicobacter pylori: pretherapeutishe resistenzlage in Deutschland (Ruhrgebiet). Z. Gastroenterol 1997; 35: 165-169.

7. Piccolomini R, di Bonaventura G, Catamo G, Carbone F, Neri M. Comparative evaluation of the $\mathrm{E}$ test, agar dilution, and broth microdilution for testing susceptibilities of Helicobacter pylori strains to 20 antimicrobial agents. J Clin Microbiol 1997; 35: $1842-1846$.

8. Canton R, de Rafael L, Boixeda D, Serano MT, GarciaGonzalez M, Baquero F. In vivo acquired resistance to fluoroquinolones in Helicobacter pylori during therapy with ciprofloxacin alone or ciprofloxacin plus amoxycillin clavulanate. In: Pajares JM, Pena AS, Malfertheiner P (eds) H. pylori and gastroduodenal pathology. Berlin, Springer-Verlag. 1993: $305-311$

9. Mégraud F. Antibiotic resistance in Helicobacter pylori infection. In: Farthing MJG, Patchett SE (eds) Helicobacter infection. Br Med Bull 1998; 54: 207-216.

10. Hartzen SH, Andersen LP, Bremmelgaard A et al. Antimicrobial susceptibility testing of 230 Helicobacter pylori strains: importance of medium, inoculum, and incubation time. Antimicrob Agents Chemother 1997; 41: 2634-2639.

11. Boyanova L, Stancheva I, Todorov D et al. Comparison of three urease tests for detection of Helicobacter pylori in gastric biopsy specimens. Eur J Gastroenterol Hepatol 1996; 8: $911-914$.

12. Fauchère JL. Validation of a disk diffusion method for macrolide susceptibility testing of Helicobacter pylori. Gut 1999; 45 Suppl III: A9.

13. Chaves S, Gadanho M, Tenreiro R, Cabrita J. Assessment of metronidazole susceptibility in Helicobacter pylori: statistical validation and error rate analysis of breakpoints determined by the disk diffusion method. J Clin Microbiol 1999; 37: $1628-1631$.

14. Midolo PD, Turnidge J, Lambert JR, Bell JM. Validation of a modified Kirby-Bauer disk diffusion method for metronidazole susceptibility testing of Helicobacter pylori. Diagn Microbiol Infect Dis 1995; 21: 135-140.

15. Xia H, Keane CT, Beattie S, O'Morain CA. Standardization of disk diffusion test and its clinical significance for susceptibility testing of metronidazole against Helicobacter pylori. Antimicrob Agents Chemother 1994, 38: 2357-2361.

16. Glupczynski Y, van der Borre C, Devaster JM, Butzler JP. Evaluation of metronidazole and clarithromycin screening agar plates for in vitro susceptibility testing of $H$. pylori. Clin Microbiol Infect 1997; 3 Suppl 2: 232.

17. National Committee for Clinical Laboratory Standards. Methods for antimicrobial susceptibility testing of anaerobic bacteria, 3rd edn. Approved standard Mll-A3. Villanova, PA, NCCLS. 1993.

18. Solnick JV. Editoral response: antibiotic resistance in Helicobacter pylori. Clin Infect Dis 1998; 27: 90-92.

19. Carbone M, Ferra MT, Cecchetti V et al. In vitro activities of new quinolones against Helicobacter pylori. Antimicrob Agents Chemother 1997; 41: 2790-2792.

20 Nakae M, Sugahara J, Sasaki H et al. [Drug-susceptibility of clinically isolated Helicobacter pylori.] Jpn J Antibiot 1998; 51: 281-285. 